\title{
General parametrization of axisymmetric black holes in metric theories of gravity
}

\author{
Roman Konoplya, ${ }^{1}$ Luciano Rezzolla, ${ }^{1,2}$ and Alexander Zhidenko ${ }^{1,3}$ \\ ${ }^{1}$ Institute for Theoretical Physics, Goethe University, Max-von-Laue-Str. 1, 60438 Frankfurt, Germany \\ ${ }^{2}$ Frankfurt Institute for Advanced Studies, Goethe-Universität, Ruth-Moufang-Str. 1, 60438 Frankfurt, Germany \\ ${ }^{3}$ Centro de Matemática, Computação e Cognição, Universidade Federal do ABC (UFABC), \\ Rua Abolição, CEP: 09210-180, Santo André, SP, Brazil
}

\begin{abstract}
Following previous work of ours in spherical symmetry, we here propose a new parametric framework to describe the spacetime of axisymmetric black holes in generic metric theories of gravity. In this case, the metric components are functions of both the radial and the polar angular coordinates, forcing a double expansion to obtain a generic axisymmetric metric expression. In particular, we use a continued-fraction expansion in terms of a compactified radial coordinate to express the radial dependence, while we exploit a Taylor expansion in terms of the cosine of the polar angle for the polar dependence. These choices lead to a superior convergence in the radial direction and to an exact limit on the equatorial plane. As a validation of our approach, we build parametrized representations of Kerr, rotating dilaton, and Einstein-dilaton-Gauss-Bonnet black holes. The match is already very good at lowest order in the expansion and improves as new orders are added. We expect a similar behavior for any stationary and axisymmetric black-hole metric.
\end{abstract}

PACS numbers: 04.50.Kd,04.70.Bw,04.25.Nx,04.30.-w,04.80.Cc

\section{INTRODUCTION}

The existence of an event horizon would be indisputable if it was obtained by the direct observation of gravitational waves from a perturbed black hole. This is because the gravitational response of a perturbed black hole would be intrinsically different from that of another putative compact object who would have essentially the same properties in terms of electromagnetic emission (see Refs. [1, 2] for the case of gravastars).

Properties of the event horizon could probably be studied through analysis of an electromagnetic spectrum of the accreting matter [3]. The radio compact source Sgr A*, which is assumed to be a supermassive black hole at the center of our Galaxy, is the best option for such investigation of the event horizon. Recent radio observations of Sgr A* achieved scales comparable to what should be the size of the event horizon [4]. In the near future, very long baseline interferometric radio observations are expected to image the so-called black-hole "shadow" [5] - the photon ring marking the surface where photons will have their smallest stable orbit [6, 7]. In addition to providing the evidence for the existence of black holes, these observations could also help in testing the no-hair theorem in general relativity [8-11] as well as testing of general relativity itself against a number of alternative theories of gravity.

Because of the large number of alternative theories of gravity and some possibility that the "true" theory is yet unknown, it is reasonable to develop a model-independent framework which parametrizes the most generic black-hole geometry through a finite number of adjustable quantities. These quantities must be chosen in such a way that they can be used to measure deviations from the general-relativistic black-hole geometry (Kerr metric) and could be estimated from the observational data [12]. This approach is similar in spirit to the parametrized post-Newtonian approach (PPN) which describes the spacetime far from the source of strong gravity [13].
One of the first such parametrizations for black holes was proposed by Johannsen and Psaltis [14], who expressed deviations from general relativity in terms of a Taylor expansion in powers of $M / r$, where $M$ and $r$ are the mass of the black hole and a generic radial coordinate. While some of the first coefficients of the expansion can be easily constrained in terms of PPN-like parameters, an infinite number remains to be determined from observations near the event horizon [14]. As pointed out by Ref. [15], this approach faces a number of difficulties:

(i) A generic metric would be described by an infinite number of roughly equally important parameters, making it difficult to isolate the dominant ones.

(ii) The parametrization can be employed to study small deviations from general relativity, but fails for essentially non-Einsteinian theories of gravity, such as, for example, Einstein-dilaton-Gauss-Bonnet (EDGB) gravity with large coupling constant [15].

(iii) The adoption of the Janis-Newman transformations [16] in the Johannsen-Psaltis parametrization [14] does not allow one to reproduce alternatives to the Kerr spacetime, for examples in dilaton or Chern-Simons modified gravity.

As a result, despite the best intentions and the wide adoption of this metric, the Johannsen-Psaltis approach basically does not seem to be a robust and generic parametrization for rotating black holes.

In a previous paper of ours [17], the solution to the above issues was proposed for arbitrary spherically symmetric black holes in metric theories of gravity. This was achieved by expressing the deviations from general relativity in terms of a continued-fraction expansion via a compactified radial coordinate defined between the event horizon and spatial infinity. The superior convergence properties of this expansion effectively reduced to a few the number of coefficients necessary 
to approximate such spherically symmetric metric to the precision that can be in principle probed with near-future observations.

In this paper we extend our approach to arbitrary axially symmetric spacetimes, describing rotating black holes in metric theories of gravity. In particular, using an asymptotic spherical coordinate system and starting from the metric functions as expressed on the equatorial plane, we perform an expansion in terms of powers of $\cos \theta$, where $\theta$ is the polar coordinate. Under appropriate choice of coordinates, leading to the Boyer-Lindquist ones in the case of zero deviations from the Kerr geometry, the expansion in $\cos \theta$ in the polar direction provides excellent convergence for known black-hole metrics, such as rotating Einstein-dilaton-Gauss-Bonnet and Johannsen-Psaltis ones.

For a number of other cases, such as Kerr, Kerr-Newman, Sen and others, the expansions in terms of powers of $\cos \theta$ converge to the corresponding exact solutions already at the second order. At the same time, when the metric components are expressed as rational functions of the radial coordinate $r$, the expansion in the radial direction follows the same behavior discussed in [17] and thus converges rapidly with a finite and small number of terms. As a result, with the approach introduced here, a number of black-hole metrics, including the Kerr spacetime, are reproduced "exactly" and in the whole space, i.e., from the event horizon out to spatial infinity.

The paper is organized as follows: In Sec. II we describe a general ansatz for axisymmetric black holes, which is further constrained by a specific coordinate choice devel- oped in Sec. III Section IV is devoted to construction of the generic parametrization, while in Sec. $\mathrm{V}$ we present the general procedure for calculation of multipole moments through the properties of the circular geodesic motion of particles around black holes. Section $\mathrm{VI}$ is devoted to construction of the parametrization for a dilaton rotating black hole and we illustrate there how the parametrization has superior convergence by computing the binding energy for exact and parametrized (at different orders) dilaton black-hole spacetimes. SectionVII presents instead the parametrization for an Einstein-dilaton-Gauss-Bonnet (EDGB) rotating black hole. Finally, Sec. VIII summarizes our results and presents our conclusions. To facilitate the use of our parametrized metrics, Appendix A provides a collection of the explicit expressions of the parametrized metrics for a Kerr, dilaton, and EDGB black hole.

\section{AXISYMMETRIC BLACK HOLES}

The general form of an axisymmetric line element allows the coordinates $t$ and $\phi$ to be along the direction selected by two Killing vectors that are timelike and spacelike, respectively. It is convenient to choose the other two spacelike coordinates, $\rho$ and $\vartheta$ to be mutually orthogonal and orthogonal to the coordinates $t$ and $\phi$, such that $(\rho, \theta, \phi)$ are spherical coordinates at spatial infinity. In this way the general form of the metric tensor for axially symmetric spacetimes can be written as

$$
d s^{2}=-\frac{f(\rho, \vartheta)-\omega^{2}(\rho, \vartheta) \sin ^{2} \vartheta}{\kappa^{2}(\rho, \vartheta)} d t^{2}-2 \omega(\rho, \vartheta) \rho \sin ^{2} \vartheta d t d \phi+\kappa^{2}(\rho, \vartheta) \rho^{2} \sin ^{2} \vartheta d \phi^{2}+\sigma(\rho, \vartheta)\left(\frac{\beta^{2}(\rho, \vartheta)}{f(\rho, \vartheta)} d \rho^{2}+\rho^{2} d \vartheta^{2}\right)
$$

where $f(\rho, \vartheta), \beta(\rho, \vartheta), \sigma(\rho, \vartheta), \kappa(\rho, \vartheta)$, and $\omega(\rho, \vartheta)$ are some dimensionless functions of the two coordinates $\rho$ and $\vartheta$.

A generic axisymmetric black-hole spacetime with line element expressed by (1) is expected to have a compact axisymmetric event horizon. Outside the horizon the introduced functions $f(\rho, \vartheta), \beta(\rho, \vartheta), \sigma(\rho, \vartheta), \kappa(\rho, \vartheta)$ are finite and positive definite, so as to avoid any "metric issue", such as singularities, closed time-like trajectories, etc.

Given the metric (1), it is easy to find that

$$
\sqrt{-g} \equiv \sqrt{-\operatorname{det}\left(g_{\mu \nu}\right)}=\beta(\rho, \vartheta) \sigma(\rho, \vartheta) \rho^{2} \sin \vartheta,
$$

and

$$
g^{\rho \rho}=\frac{f(\rho, \vartheta)}{\beta^{2}(\rho, \vartheta)}
$$

From the latter expression we conclude that the Killing horizon (i.e., the surface defined by the null Killing vector) is given by

$$
f(\rho, \vartheta)=0
$$

while the ergoregion is instead defined as

$$
0<f(\rho, \vartheta)<\omega^{2}(\rho, \vartheta) \sin ^{2} \vartheta .
$$

Since $\omega(\rho, \vartheta)$ is finite at the Killing horizon, the ergosphere always touches the horizon at the poles $\vartheta=0, \pi$.

Further we shall consider the rotating dilaton black hole (or Kerr-Sen) black hole [18] or axion-dilaton black hole with zero Newman-Unti-Tamburino (NUT) charge [19]) as one of the examples. The above five functions for this black hole are

$$
\begin{aligned}
f(\rho, \vartheta) & =\frac{\rho^{2}-2 \mu \rho+a^{2}}{\rho^{2}}, \\
\beta(\rho, \vartheta) & =1, \\
\sigma(\rho, \vartheta) & =\frac{\rho^{2}+2 b \rho+a^{2} \cos ^{2} \vartheta}{\rho^{2}},
\end{aligned}
$$

$\kappa^{2}(\rho, \vartheta)=\frac{\left(\rho^{2}+2 b \rho+a^{2}\right)^{2}-a^{2} \sin ^{2} \vartheta\left(\rho^{2}-2 \mu \rho+a^{2}\right)}{\rho^{2}\left(\rho^{2}+2 b \rho+a^{2} \cos ^{2} \vartheta\right)}$,

$$
\omega(\rho, \vartheta)=\frac{2(\mu+b) a}{\rho^{2}+2 b \rho+a^{2} \cos ^{2} \vartheta},
$$


where $a \equiv J / M$ and $b$ are parameters of rotation and dilaton respectively, and $\mu \equiv M-b$, where $M$ is the black-hole mass. Clearly, when $b=0$, the above metric components reduce to those of the Kerr spacetime in Boyer-Lindquist coordinates $(\rho, \theta, \phi)$.

\section{COORDINATE CHOICES AND EXPANSION IN THE POLAR DIRECTION}

The choice of functions $f(\rho, \vartheta), \beta(\rho, \vartheta), \sigma(\rho, \vartheta), \kappa(\rho, \vartheta)$, and $\omega(\rho, \vartheta)$ for a given axisymmetric black hole is not unique. Instead of $\rho$ and $\vartheta$ we could in fact choose another couple of coordinates, which are also mutually orthogonal and orthogonal to the coordinates $t$ and $\phi$. Thus, at this stage the coordinates are not fully fixed: a single black hole can be represented by a number of different systems of coordinates within the same initial ansatz (1). In the end this would produce a nonunique parametrization, when the same black hole could be described by a number of different sets of parameters. To avoid such a degeneracy, we will here impose further conditions which fix the coordinates fully.

In order to describe how to attain such a unique coordinate choice, let us first consider transformations from the initial coordinates $(\rho, \vartheta)$ to the new ones $(r, \theta)$, such that the new line element is expressed in terms of five metric functions $N(r, \theta)$, $W(r, \theta), K(r, \theta), B(r, \theta), \Sigma(r, \theta)$, and has the following form

$d s^{2}=-\frac{N^{2}(r, \theta)-W^{2}(r, \theta) \sin ^{2} \theta}{K^{2}(r, \theta)} d t^{2}-2 W(r, \theta) r \sin ^{2} \theta d t d \phi+K^{2}(r, \theta) r^{2} \sin ^{2} \theta d \phi^{2}+\Sigma(r, \theta)\left(\frac{B^{2}(r, \theta)}{N^{2}(r, \theta)} d r^{2}+r^{2} d \theta^{2}\right)$.

Comparing the inverse metric components of (1) and (7) we find the following relations

$$
\begin{aligned}
N^{2}(r, \theta) r^{2} \sin ^{2} \theta & =f(\rho, \vartheta) \rho^{2} \sin ^{2} \vartheta, \\
W(r, \theta) r \sin ^{2} \theta & =\omega(\rho, \vartheta) \rho \sin ^{2} \vartheta, \\
K^{2}(r, \theta) r^{2} \sin ^{2} \theta & =\kappa^{2}(\rho, \vartheta) \rho^{2} \sin ^{2} \vartheta, \\
\frac{N^{2}(r, \theta)}{\Sigma(r, \theta) B^{2}(r, \theta)} & =\frac{1}{\sigma(\rho, \vartheta)}\left(\frac{f(\rho, \vartheta)}{\beta^{2}(\rho, \vartheta)} \frac{\partial r}{\partial \rho} \frac{\partial r}{\partial \rho}+\frac{1}{\rho^{2}} \frac{\partial r}{\partial \vartheta} \frac{\partial r}{\partial \vartheta}\right),
\end{aligned}
$$$$
\frac{1}{\Sigma(r, \theta) r^{2}}=\frac{1}{\sigma(\rho, \vartheta)}\left(\frac{f(\rho, \vartheta)}{\beta^{2}(\rho, \vartheta)} \frac{\partial \theta}{\partial \rho} \frac{\partial \theta}{\partial \rho}+\frac{1}{\rho^{2}} \frac{\partial \theta}{\partial \vartheta} \frac{\partial \theta}{\partial \vartheta}\right)
$$$$
0=\frac{f(\rho, \vartheta)}{\beta^{2}(\rho, \vartheta)} \frac{\partial r}{\partial \rho} \frac{\partial \theta}{\partial \rho}+\frac{1}{\rho^{2}} \frac{\partial r}{\partial \vartheta} \frac{\partial \theta}{\partial \vartheta} .
$$

The last two equations define the relation between the coordinates $(r, \theta)$ and $(\rho, \vartheta)$. Once one finds $r$ and $\theta$ as functions of $\rho$ and $\vartheta$ through Eqs. (8e), 8f , it is possible to find the functions $N(r, \theta), W(r, \theta), K(r, \theta)$, and $B(r, \theta)$ from Eqs. (8a), 8b, 8c), and (8d), respectively.

Next, we assume that the functions $f(\rho, \vartheta), \beta(\rho, \vartheta)$, $\sigma(\rho, \vartheta), \kappa(\rho, \vartheta)$, and $\omega(\rho, \vartheta)$ are known as series expansion in terms of small parameter $\mathcal{Y}$ measuring the distance from the equatorial plane, i.e., defined as $\mathcal{Y} \equiv \cos \vartheta$. Hence, we have

$$
\begin{aligned}
f & =f_{0}(\rho)+f_{1}(\rho) \mathcal{Y}+f_{2}(\rho) \mathcal{Y}^{2}+\mathcal{O}\left(\mathcal{Y}^{3}\right) \\
\beta & =\beta_{0}(\rho)+\beta_{1}(\rho) \mathcal{Y}+\beta_{2}(\rho) \mathcal{Y}^{2}+\mathcal{O}\left(\mathcal{Y}^{3}\right) \\
\sigma & =\sigma_{0}(\rho)+\sigma_{1}(\rho) \mathcal{Y}+\sigma_{2}(\rho) \mathcal{Y}^{2}+\mathcal{O}\left(\mathcal{Y}^{3}\right) \\
\kappa & =\kappa_{0}(\rho)+\kappa_{1}(\rho) \mathcal{Y}+\kappa_{2}(\rho) \mathcal{Y}^{2}+\mathcal{O}\left(\mathcal{Y}^{3}\right) \\
\omega & =\omega_{0}(\rho)+\omega_{1}(\rho) \mathcal{Y}+\omega_{2}(\rho) \mathcal{Y}^{2}+\mathcal{O}\left(\mathcal{Y}^{3}\right)
\end{aligned}
$$

where the coefficients in the expansion, i.e., $f_{i}, \beta_{i}, \sigma_{i}, \ldots$ are functions of the radial coordinate only.
Introducing now the small parameter $y \equiv \cos \theta$, we can find the corresponding new transformation $(\mathcal{Y}, \rho) \rightarrow(y, r)$ as a series expansion in terms of $\mathcal{Y}$, namely

$$
\begin{aligned}
y & =\mathcal{Y}\left(1+\alpha_{0}(\rho)\right)\left[1+\alpha_{1}(\rho) \mathcal{Y}+\alpha_{2}(\rho) \mathcal{Y}^{2}+\mathcal{O}\left(\mathcal{Y}^{2}\right)\right] \\
r^{2} & =\rho^{2}\left(1+\zeta_{0}(\rho)\right)\left[1+\zeta_{1}(\rho) \mathcal{Y}+\zeta_{2}(\rho) \mathcal{Y}^{2}+\mathcal{O}\left(\mathcal{Y}^{2}\right)\right]
\end{aligned}
$$

A few remarks should be made here. First, if all the coefficients $\alpha_{i}(\rho)=0$, then $\theta=\vartheta$, that is, the two polar coordinates are identical. Second, if all coefficients $\zeta_{i}(\rho)=0$, then also the two radial coordinates are identical, i.e., $r=\rho$. Finally and most important, we have here decided to perform a generic expansion in powers of the small parameter $\cos \theta$. This choice allows us to consider black holes that are not reflection symmetric across the equatorial plane. This is admittedly a bizarre possibility, but one we want to preserve for generality. In practice, any representation of an astrophysically reasonable black hole would require also the reflection symmetry across the equatorial plane, thus limiting the expansion to even powers of $\cos \theta$. Indeed this is what we will do when considering the parametrization of Kerr, dilaton, and EDGB black holes in the following sections.

Without loss of generality we can assume that there are observers at spatial infinity that are able to measure the angular momentum $J \equiv A M$ and mass $M$ of the black hole. With this assumption, we are then able to define the coordinates $r$ and $\theta$ unambiguous.

More specifically, we first need to fix one of the five metric functions $N(r, \theta), W(r, \theta), K(r, \theta), B(r, \theta), \Sigma(r, \theta)$. Out of the five possible choices, we prefer to fix the function $\Sigma(r, \theta)$ as

$$
\Sigma(r, \theta)=1+\frac{A^{2} y^{2}}{r^{2}}=1+\frac{A^{2} \cos ^{2} \theta}{r^{2}},
$$

since this allows us to reproduce the Boyer-Lindquist coordi- 
nates for the case of a Kerr black hole. This choice is also compatible with asymptotic behavior of the asymptotically flat and axially symmetric metric of a rotating body in the Boyer-Lindquist coordinates

$$
\begin{aligned}
d s^{2} \approx & -\left(1-\frac{2 M}{r}\right) d t^{2}-\frac{4 M A \sin ^{2} \theta}{r} d t d \phi+d r^{2} \\
& +r^{2}\left(d \theta^{2}+\sin ^{2} \theta d \phi^{2}\right) .
\end{aligned}
$$

In an astrophysically realistic context, at large distance the gravitational field of a rotating object of any kind should be essentially Newtonian, thus, hiding the details related to the event horizon of an isolated black hole and regime of strong gravity. The addition of extra fields to the black-hole spacetime, e.g., scalar or electromagnetic, would certainly change the asymptotic behavior. Yet, backreaction of such fields onto the background geometry is expected to be negligibly small for astrophysical black holes, thereby, allowing us to consider an isolated black hole as in vacuum.

A more careful analysis reveals that the condition (12) is still insufficient to fix completely the freedom of the coordinate choice. The reason for this is that using Eqs. (8e and (8f) it is possible to obtain different series for $y$ and $r$ for each different choice of $\alpha_{0}(\rho)$. Thus, the function $\alpha_{0}(\rho)$ must be fixed in order to achieve the uniqueness of the coordinate transformations (10). The natural way to fix $\alpha_{0}(\rho)$ is to choose an additional condition for the new line element in the equatorial plane in such a way that for the zero rotation we reproduce spherical coordinates. This can be done in various ways, but we here choose to impose a condition on the function $K$ on the equatorial plane. More specifically, we first observe that, multiplying (8b) by any constant $C$ and adding $(8 \mathrm{c})$, we obtain

$$
\left(K^{2}+\frac{C}{r} W\right) r^{2} \sin ^{2} \theta=\left(\kappa^{2}+\frac{C}{r} \omega\right) \rho^{2} \sin ^{2} \vartheta,
$$

which, in turn, allows us to impose that

$$
\left(K^{2}-\frac{A}{r} W\right)=1+\frac{A^{2}}{r^{2}}+\mathcal{O}(y) .
$$

It is not difficult to verify that the Boyer-Lindquist coordinates fulfill the above condition. This gives us the unambiguous coordinate choice for $r$ and $\theta$.

In summary, in order to transform unambiguously any given coordinates $(\rho, \vartheta)$ to the new coordinates $(r, \theta)$ we need to

1. define the rotation parameter $A \equiv J / M$, where $J$ is the total angular momentum and $M$ is the asymptotic mass of the spacetime 1 ;

\footnotetext{
${ }^{1}$ The constant $M$ could be associated with the Arnowitt-Deser-Misner (ADM) mass at spatial infinity if such a quantity can be properly defined. However, because we are not limiting ourselves to asymptotically flat spacetimes where such a quantity is defined, we here consider the more general case in which astronomical observations at large distances from the event horizon but not at spatial infinity can be exploited to measure the constant $M$.
}

2. choose $r$ and $\theta$ to be mutually orthogonal and orthogonal to the coordinates $t$ and $\phi$;

3. impose that the metric functions satisfy the conditions (12) and 15, namely

$$
\begin{aligned}
\Sigma(r, \theta) & =1+\frac{A^{2}}{r^{2}} \cos ^{2} \theta, \\
K^{2}\left(r, \frac{\pi}{2}\right)-\frac{A}{r} W\left(r, \frac{\pi}{2}\right) & =1+\frac{A^{2}}{r^{2}} .
\end{aligned}
$$

The latter condition in fact allows us to fix the function $\alpha_{0}(\rho)$ as

$$
1+\alpha_{0}(\rho)=\sqrt{\frac{\sigma_{0}(\rho)}{\kappa_{0}^{2}(\rho)-A \omega_{0}(\rho) / \rho-A^{2} / \rho^{2}}} .
$$

so that, from Eqs. (8e) and (8f), we finally find

$$
\begin{aligned}
y & =\mathcal{Y}\left(1+\alpha_{0}(\rho)\right)\left[1+\frac{\sigma_{1}(\rho)}{4 \sigma_{0}(\rho)} \mathcal{Y}+\mathcal{O}\left(\mathcal{Y}^{2}\right)\right], \\
r^{2} & =\frac{\rho^{2} \sigma_{0}(\rho)}{\left(1+\alpha_{0}(\rho)\right)^{2}}+\mathcal{O}\left(\mathcal{Y}^{2}\right)
\end{aligned}
$$

In analogy with what was done for the expressions (9), we can now invert the series (19) to find the functions $\mathcal{Y}(r, y)$ and $\rho(r, y)$ and thus express the metric functions $N^{2}, W, K^{2}$, and $B^{2}$ in the line element (7) as a series in terms of the new variable $y$.

\section{PARAMETRIZATION FOR AXISYMMETRIC BLACK HOLES}

In order to obtain the proper asymptotic behavior of the newly introduced metric function we need to ensure they satisfy the following behavior at large distances, namely that for $r \gg 1$

$$
\begin{aligned}
N^{2}(r, \theta) & =1-\frac{2 M}{r}+\mathcal{O}\left(\frac{1}{r^{2}}\right), \\
B(r, \theta) & =1+\mathcal{O}\left(\frac{1}{r}\right), \\
W(r, \theta) & =\mathcal{O}\left(\frac{1}{r^{2}}\right), \\
K^{2}(r, \theta) & =1+\mathcal{O}\left(\frac{1}{r^{2}}\right),
\end{aligned}
$$

where $M$ is a constant to be read at a large distance.

These conditions on the metric functions obviously imply that the metric is asymptotically flat and spherically symmetric at large distance from the black hole. In principle, these conditions could be violated in a cosmology admitting violation of isotropy, such as the Einstein-Aether theory. Yet, it is evident that local physical processes around black holes cannot be influenced by such cosmological factors while the coupling constant of new interactions (be it vector Aether or any 
other field) is negligibly small for observations of localized processes.

Now that we have performed the coordinate transformations (19) and all the functions are obtained as series expansion in terms of the "angular" variable $y=\cos \theta$, it is necessary to introduce the parametrization for the coefficients of the series which are functions of the radial coordinate only. Also in this case there are several different ways in which this can be accomplished. Here, however, we will follow the powerful approach already employed in [17] for the parametrization of a generic black-hole metric in spherical symmetry. More specifically, we first introduce the compact coordinate radial

$$
x=1-\frac{r_{0}}{r},
$$

where $r_{0}$ is the black-hole horizon radius in the equatorial plane; i.e., $r_{0}$ is the largest solution of the equation

$$
N^{2}(r, \pi / 2)=0 \text {. }
$$

Clearly $x \in[0,1]$, with $x=0$ at the black-hole horizon on the equatorial plane (i.e., $y=0$ ) and $x=1$ at spatial infinity.

Second, we consider the following expansions in terms of the new compact coordinate $x$,

$$
\begin{aligned}
N^{2} & =x A_{0}(x)+\sum_{i=1}^{\infty} A_{i}(x) y^{i}, \\
B & =1+\sum_{i=0}^{\infty} B_{i}(x) y^{i}, \\
W & =\sum_{i=0}^{\infty} \frac{W_{i}(x) y^{i}}{\Sigma} \\
K^{2}-\frac{A W}{r} & =1+\sum_{i=0}^{\infty} \frac{K_{i}(x) y^{i}}{\Sigma}
\end{aligned}
$$

where from 16

$$
\Sigma=1+\frac{A^{2}}{r_{0}^{2}}(1-x)^{2} y^{2} .
$$

Our coordinate choice (15) then fixes $K_{0}$ to be

$$
K_{0}(x)=(1-x)^{2} \frac{A^{2}}{r_{0}^{2}} .
$$

Third, in order to satisfy the required asymptotic behavior (20), we define

$$
\begin{gathered}
B_{i}(x)=b_{i 0}(1-x)+\tilde{B}_{i}(x)(1-x)^{2} \\
W_{i}(x)=w_{i 0}(1-x)^{2}+\tilde{W}_{i}(x)(1-x)^{3} \\
K_{i}(x)=k_{i 0}(1-x)^{2}+\tilde{K}_{i}(x)(1-x)^{3} \\
\begin{aligned}
A_{0}(x)= & 1-\epsilon_{0}(1-x)+\left(a_{00}-\epsilon_{0}+k_{00}\right)(1-x)^{2} \\
& +\tilde{A}_{0}(x)(1-x)^{3} \\
A_{i>0}(x)= & K_{i}(x)+\epsilon_{i}(1-x)^{2}+a_{i 0}(1-x)^{3}+ \\
& +\tilde{A}_{i}(x)(1-x)^{4}
\end{aligned}
\end{gathered}
$$

Note that the coefficients $\epsilon_{i}, a_{i 0}, b_{i 0}, w_{i 0}, k_{i 0}$ for $i=$ $0,1,2,3 \ldots$ are fixed in such a way that the expansion (26) matches desired asymptotic behavior near spatial infinity (i.e., $x=1$ ). To make it astrophysically meaningful, the latter should be expressed in the terms of the PPN expansion.

Since the tilted functions $\tilde{A}_{i}(x), \tilde{B}_{i}(x), \tilde{W}_{i}(x)$, and $\tilde{K}_{i}(x)$ describe the black-hole metric near its horizon, we here express them in close analogy with what was already done in [17], and thus parametrize them in terms of Padé approximants in the form of continued fraction

$$
\begin{aligned}
\tilde{A}_{i}(x) & =\frac{a_{i 1}}{1+\frac{a_{i 2} x}{1+\frac{a_{i 3} x}{1+\ldots}}}, \\
\tilde{B}_{i}(x) & =\frac{b_{i 1}}{1+\frac{b_{i 2} x}{1+\frac{b_{i 3} x}{1+\ldots}}}, \\
\tilde{W}_{i}(x) & =\frac{w_{i 1}}{1+\frac{w_{i 2} x}{1+\frac{w_{i 3} x}{1+\ldots}}}, \\
\tilde{K}_{i}(x) & =\frac{k_{i 1}}{1+\frac{k_{i 2} x}{1+\frac{k_{i 3} x}{1+\ldots}}},
\end{aligned}
$$

where $a_{i j}, b_{i j}, w_{i j}, k_{i j}$ for $i=0,1,2,3 \ldots, j=1,2,3 \ldots$ are fixed via a comparison of the series expansions of the metric functions near the black-hole horizon (i.e., $x=0$ ) with their exact analytic expressions or of numerical data when an exact solution cannot be obtained analytically.

To recap: Two different sets of coefficients appear in the approach proposed here for the parametrization of a generic stationary and axisymmetric black-hole metric. The first set is given by the coefficients $a_{i 0}, b_{i 0}, w_{i 0}, k_{i 0}, \epsilon_{i}(i=1,2,3 \ldots)$, which are fixed by spacetime behavior in the asymptotic region, i.e., $x \rightarrow 1^{-}$. The second set is instead given by the coefficients $a_{i j}, b_{i j}, w_{i j}, k_{i j}(j=1,2,3 \ldots)$, which are fixed by the geometry of the black hole near its horizon, i.e., $x \rightarrow 0^{+}$. Needless to say, such a separation of parameters on "near horizon" and "asymptotic" is essential for the comparison of the theoretical predictions with the observational data obtained, either in the far region (e.g., values of PPN parameters) or near the black hole (e.g., study of accretion flows, black hole's shadows, etc.). It should also be noted that the contribution of $K_{i}(x)$ in the definition of $A_{i>0}(x)$ allows us to define the asymptotic parameters $\epsilon_{i}$ and $a_{i 0}$, by comparing the asymptotic expansions of the metric component $g_{t t}$. Without this contribution of $K_{i}(x)$, we would not be able to separate the parameters $a_{i 0}$ and $k_{i j}$ into, "asymptotic" and "nearhorizon" ones, respectively.

It is important to discuss now some essential properties of the continued fraction used in the Padé approximation in (27), namely, the number of terms $N$ appearing in the continued fractions. To this scope we will consider three different cases.

The first case is the one for which the tilted functions $\tilde{A}_{i}(x)$, $\tilde{B}_{i}(x), \tilde{W}_{i}(x)$, and $\tilde{K}_{i}(x)$ are fractions of two polynomials of 
$r$, as it happens, for example, for any of these functions in the case of the Kerr solution. In this case, then, the corresponding expansion (27) contains a finite and small number of terms. In other words, there exist a number $N>0$, such that $a_{i N}=0$, or $b_{i N}=0$, or $w_{i N}=0$, or $k_{i N}=0$, respectively. In this case, all higher-order terms are obviously not necessary and we refer to this representation as "exact", in the sense that the corresponding metric can be reproduced analytically with only a finite number of coefficients.

The second case is for when the metric functions $N(r, \theta)$, $W(r, \theta), K(r, \theta)$, and $B(r, \theta)$ in (7) are not rational functions of $r$. In this case, then, some or all of the expansions 27) contain an infinite number of coefficients, i.e., $N=\infty$. Finally, the third case is for when even though $N$ is finite, there is no guarantee that an "exact" representation will be achieved with a small number of coefficients. As a result, in both of these last two cases (i.e., of finite but large and infinite $N$ ), the metric representation will be only approximate and limited to the first $n<N$ terms, setting the $n$-th equal to zero. We will refer to such approximated metrics obtained by truncating at the $n$-th term as the "approximation of the $(n-1)$-th order".

As a corollary to the previous remark we also note that the $n$-th coefficient cannot always be set equal to zero in a consistent manner. In some cases, in fact, setting a particular coefficient to zero could imply the divergence of the truncated continued fractions (27) for the corresponding tilted function for some value of $r$ outside the event horizon. To solve this problem within the approximation of the $(n-1)$-th order, one should set the $(n+1)$-th coefficient equal to zero, and choose an arbitrary value for the $n$-th coefficient, so that the denominator remains positive definite for all values of the radial coordinate outside the event horizon (i.e., for $x>0$ ). For convenience, in such cases we will take the value of the $n$-th coefficient to be equal to unity and will refer to these cases as approximations of $(n-1)$-th order as well.

As a final remark we note that not all of the parameters so far, i.e., $a_{i j}, b_{i j}, w_{i j}$, and $k_{i j}$, are effectively independent. This is because one of the infinite number of functions, i.e., $A_{i}(x), B_{i}(x), W_{i}(x)$, and $K_{i}(x)$, must be fixed by a coordinate choice. We have here used the condition (25), so that, taking into account the continued fractions 27, yields

$$
k_{00} \equiv \frac{A^{2}}{r_{0}^{2}}, \quad k_{01} \equiv 0
$$

In the next section, on the other hand, we will not assume any particular coordinate condition of the type (28), leaving a possibility to fix it in any alternative way.

\section{A. Asymptotic properties}

Following the discussion on the asymptotic properties of the parametrized metric made with expression (13), we can deduce the following asymptotic behavior of the line element
(7)

$$
\begin{aligned}
\frac{N^{2}(r, \theta)-}{K^{2}(r, \theta) \sin ^{2} \theta} & = \\
& =\frac{N^{2}(r, \theta)}{K^{2}(r, \theta)-A W(r, \theta) / r}+\mathcal{O}\left(\frac{1}{r^{3}}\right) \\
& =1-\frac{2 M}{r}+(\beta-\gamma) \frac{2 M}{r^{2}}+\mathcal{O}\left(\frac{1}{r^{3}}\right), \\
W(r, \theta) & =\frac{2 J}{r^{2}}+\mathcal{O}\left(\frac{1}{r^{3}}\right)=\frac{2 M A}{r^{2}}+\mathcal{O}\left(\frac{1}{r^{3}}\right), \\
\frac{B^{2}(r, \theta)}{N^{2}(r, \theta)} & =1+\gamma \frac{2 M}{r}+\mathcal{O}\left(\frac{1}{r^{2}}\right) \\
K^{2}(r, \theta) & =1+\mathcal{O}\left(\frac{1}{r^{2}}\right) .
\end{aligned}
$$

Note that in deriving expressions (29) we have also introduced the PPN parameters $\beta$ and $\gamma$ [13] and assumed a reflection symmetry across the equatorial plane, i.e., the functions in (23) are taken to depend only on $y^{2}(i=0,2,4 \ldots)$. Furthermore, adopting the classification made by Thorne in Ref. [20], metrics of this type are referred to as "Cartesian and mass centered to order 0" (ACMC-0). Clearly, using expressions 29], it is possible to read off the mass $M$ and angular momentum $J$ of the spacetime. We note that although the asymptotic behavior we have chosen is well motivated from an astrophysical point of view, our approach is not limited by any particular choice of asymptotic behavior and can, in principle, be constructed also for axisymmetric black holes having different asymptotic constraints.

Next, for a metric with the asymptotic behavior given by expressions (29), we find that

$$
\begin{aligned}
\epsilon_{0} & =\frac{2 M-r_{0}}{r_{0}}, \\
a_{00} & =(\beta-\gamma) \frac{2 M^{2}}{r_{0}^{2}}=\frac{(\beta-\gamma)\left(1+\epsilon_{0}\right)^{2}}{2}, \\
b_{00} & =(\gamma-1) \frac{M}{r_{0}}=\frac{(\gamma-1)\left(1+\epsilon_{0}\right)}{2} \\
w_{00} & =\frac{2 J}{r_{0}^{2}}=\frac{J}{M^{2}} \frac{\left(1+\epsilon_{0}\right)^{2}}{2} \\
\epsilon_{i} & =0=b_{i 0}=w_{i 0}, \quad i>0 .
\end{aligned}
$$

The asymptotic parameters, $a_{i 0}$ and $k_{i 0}$ for $i>0$, are not fixed only by $M, J, \beta$, and $\gamma$. In particular, the parameter $a_{20}$ contains also information on the quadrupole moment of the black hole, which cannot be read off from the asymptotic expansion. This is because the metric with components (26) and with the parameters fixed by expressions (30), is not of type ACMC- 1 unless $b_{i 1}=0$ and $k_{i 0}=0$ for all $i>0$. In this latter case, $a_{20}$ is related to the multipole $I^{20}$ introduced by Thorne in [20]; in particular, using expression (11.4a) of 
[20], we can read off the value of $a_{20}$ as

$$
I^{20}=-\frac{4}{3} \sqrt{\frac{4 \pi}{15}} a_{20} r_{0}^{3} .
$$

Note that since $b_{i 1}$ are parameters fixed near the black-hole horizon, we are unable to find a general transformation from (7) to an ACMC-1 type of metric and cannot, therefore, express $I^{20}$ only in terms of the asymptotic parameters. Nevertheless, as we will show in the next section, $a_{i 0}$ and $k_{i 0}$ can be related to observable quantities in a way similar to what was done for the Geroch-Hansen quadrupole moment of the black hole [21]. In particular, we will compare the orbital-plane precession frequency with the formula derived by Ryan [23], thus obtaining a definition of the quadrupole moment through the asymptotic parameters only.

\section{CIRCULAR GEODESIC IN THE EQUATORIAL PLANE AND MULTIPOLE MOMENTS}

Following [23], we consider a circular geodesic motion in the equatorial plane, i.e., with

$$
\frac{d r}{d t}=0, \quad \theta=\frac{\pi}{2}
$$

and with orbital frequency

$$
\Omega \equiv \frac{d \phi}{d t}=\frac{-g_{t \phi, r}+\sqrt{g_{t \phi, r}^{2}-g_{t t, r} g_{\phi \phi, r}}}{g_{\phi \phi, r}}
$$

where we use a comma to indicate a partial derivative. The energy per unit mass is then given by (see, e.g., [22])

$$
\frac{E}{m}=\frac{-g_{t t}-g_{t \phi} \Omega}{\sqrt{-g_{t t}-2 g_{t \phi} \Omega-g_{\phi \phi} \Omega^{2}}},
$$

and, after using expression (33), it can be expressed as a function of $\Omega$ only. After replacing in (34) the parametrized expressions for the metric functions, we can derive an expression for the energy per logarithmic interval of frequency in terms of the dimensionless variable $v \equiv(\Omega M)^{1 / 3}$

$$
\begin{aligned}
\frac{\Delta E}{m}=-\frac{1}{m} \frac{d E}{d \log \Omega}=-\frac{v}{3 m} \frac{d E}{d v}=\frac{v^{2}}{3}+v^{4}\left(-\frac{1}{2}+\frac{8 a_{00}}{9\left(1+\epsilon_{0}\right)^{2}}\right)+v^{5} \frac{40 w_{00}}{9\left(1+\epsilon_{0}\right)^{2}}+ \\
v^{6}\left[-\frac{27}{8}+\frac{6 a_{00}+4 k_{00}-4 w_{00} A / M}{\left(1+\epsilon_{0}\right)^{2}}+\frac{16 a_{00}^{2}}{3\left(1+\epsilon_{0}\right)^{4}}+8 \frac{\epsilon_{0}-a_{00}-k_{00}+\tilde{A}_{0}(1)-\tilde{K}_{0}(1)}{\left(1+\epsilon_{0}\right)^{3}}\right]+\mathcal{O}\left(v^{7}\right),
\end{aligned}
$$

where

$$
\begin{gathered}
\tilde{A}_{0}(1)=\frac{a_{01}}{1+\frac{a_{02}}{1+\frac{a_{03}}{1+\ldots}}}, \\
\tilde{K}_{0}(1)=\frac{k_{01}}{1+\frac{k_{02}}{1+\frac{k_{03}}{1+\ldots}}} .
\end{gathered}
$$

In a similar way, we can obtain a series expansion of the periastron precession frequency $\Omega_{r}$ and orbital-plane precession frequency $\Omega_{\theta}$, defined as [23]

$$
\begin{aligned}
& \Omega_{r} \equiv \Omega-\sqrt{-\frac{A_{E}^{2} G_{\phi \phi, r r}+2 A_{E} A_{L} G_{t \phi, r r}+A_{L}^{2} G_{t t, r r}}{2 g_{r r}}}, \\
& \Omega_{\theta} \equiv \Omega-\sqrt{-\frac{A_{E}^{2} G_{\phi \phi, \theta \theta}+2 A_{E} A_{L} G_{t \phi, \theta \theta}+A_{L}^{2} G_{t t, \theta \theta}}{2 g_{\theta \theta}}},
\end{aligned}
$$

where

$$
\begin{aligned}
A_{E} & \equiv-g_{t t}-g_{t \phi} \Omega=\frac{N^{2}}{K^{2}}+W \sin ^{2} \theta\left(r \Omega-\frac{W}{K^{2}}\right) \\
A_{L} & \equiv g_{t \phi}+g_{\phi \phi} \Omega=r K^{2} \sin ^{2} \theta\left(r \Omega-\frac{W}{K^{2}}\right), \\
G_{\alpha \beta} & \equiv \frac{g_{\alpha \beta}}{g_{t \phi}^{2}-g_{t t} g_{\phi \phi}}=\frac{g_{\alpha \beta}}{N^{2} r^{2} \sin ^{2} \theta}, \quad \alpha, \beta=t, \phi .
\end{aligned}
$$

Collecting terms, we can express the normalized periastron precession and orbital-plane precession frequencies respectively as 


$$
\begin{aligned}
\frac{\Omega_{\theta}}{\Omega}= & -v^{2} \frac{2 \epsilon_{2}}{\left(\epsilon_{0}+1\right)^{2}}+v^{3} \frac{4\left(w_{00}-w_{20}\right)}{\left(\epsilon_{0}+1\right)^{2}}+v^{4}\left[-\frac{2\left(k_{20}+k_{00}-w_{20} A / M\right)}{\left(\epsilon_{0}+1\right)^{2}}-\frac{4 a_{20}}{\left(\epsilon_{0}+1\right)^{3}}+\frac{6 \epsilon_{2}^{2}-32 \epsilon_{2} a_{00}}{3\left(\epsilon_{0}+1\right)^{4}}\right]+\mathcal{O}\left(v^{5}\right), \\
\frac{\Omega_{r}}{\Omega}= & v^{2}\left[3-\frac{2 a_{00}}{\left(\epsilon_{0}+1\right)^{2}}+\frac{b_{00}}{2\left(\epsilon_{0}+1\right)}\right]-\frac{8 w_{00} v^{3}}{\left(\epsilon_{0}+1\right)^{2}}+v^{4}\left[\frac{9}{2}-\frac{3 b_{00}}{2\left(\epsilon_{0}+1\right)}-\frac{8 a_{00}+6 k_{00}-6 w_{00} A / M}{\left(\epsilon_{0}+1\right)^{2}}\right. \\
& \left.-\frac{5 b_{00}^{2}}{8\left(\epsilon_{0}+1\right)^{2}}+\frac{5 b_{00} a_{00}}{3\left(\epsilon_{0}+1\right)^{3}}-\frac{26 a_{00}^{2}}{3\left(\epsilon_{0}+1\right)^{4}}+\frac{12\left(a_{00}+k_{00}+k_{01}-\epsilon_{0}-\tilde{A}_{0}(1)\right)}{\left(\epsilon_{0}+1\right)^{3}}+\frac{\tilde{B}_{0}(1)}{\left(\epsilon_{0}+1\right)^{2}}\right]+\mathcal{O}\left(v^{5}\right),
\end{aligned}
$$

where

$$
\tilde{B}_{0}(1)=\frac{b_{01}}{1+\frac{b_{02}}{1+\frac{b_{03}}{1+\ldots}}}
$$

We can now compare expressions (43) and (44) with the corresponding ones obtained by Ryan in general relativity [23], i.e.,

$$
\begin{aligned}
& \frac{\Delta E}{m}=\frac{v^{2}}{3}-\frac{v^{4}}{2}+v^{5} \frac{20 S_{1}}{9 M_{0}^{2}}+v^{6}\left(-\frac{27}{8}+\frac{M_{2}}{M_{0}^{3}}\right)+\mathcal{O}\left(v^{7}\right), \\
& \frac{\Omega_{\theta}}{\Omega}=v^{3} \frac{2 S_{1}}{M_{0}^{2}}+v^{4} \frac{3 M_{2}}{2 M_{0}^{3}}+\mathcal{O}\left(v^{5}\right) \\
& \frac{\Omega_{r}}{\Omega}=3 v^{2}-v^{3} \frac{4 S_{1}}{M_{0}^{2}}+v^{4}\left(\frac{9}{2}-\frac{3 M_{2}}{2 M_{0}^{3}}\right)+\mathcal{O}\left(v^{5}\right)
\end{aligned}
$$

where $M_{0}=M, S_{1}=J$, and $M_{2}=Q$ are the first three Geroch-Hansen multipole moments: the ADM mass, the angular momentum and the quadrupole moment, respectively.

Bearing in mind that

$$
w_{00}=\frac{2 J}{r_{0}}, \quad \Longleftrightarrow \quad \frac{J}{M^{2}}=\frac{2 w_{00}}{\left(\epsilon_{0}+1\right)^{2}},
$$

we obtain [cf. Eqs. (30) for $\beta=\gamma=1$ ]

$$
\begin{gathered}
\epsilon_{2}=0=w_{20}=a_{00}=b_{00}, \\
-\frac{3 Q}{2 M^{3}}=\frac{2\left(k_{20}+k_{00}\right)}{\left(\epsilon_{0}+1\right)^{2}}+\frac{4 a_{20}}{\left(\epsilon_{0}+1\right)^{3}} .
\end{gathered}
$$

The expression for the quadrupole moment $Q$ through the values of the coefficients as fixed at spatial infinity can be obtained only after measuring the orbital-plane precession frequency (43). Note that in the case in which $\epsilon_{2}=0$, such a frequency (43) does not depend on the PPN parameters that are related to the coefficient $a_{00}$ [the fifth term in Eq. (43) is automatically zero in this case]. This property suggests that the expression (49) derived in general relativity remains the same also for non-Einsteinian theories. Indeed, as we will discuss in Sec. VII it provides the correct answer for the Einsteindilaton-Gauss-Bonnet black holes. On the other hand, the expressions for the energy per logarithmic frequency interval (35) and for the orbital-plane precession frequency (44), allow us to obtain those higher-order PPN parameters that are encoded, within our formalism, in the values of the coefficients $\tilde{A}_{0}(1), \tilde{B}_{0}(1)$, and $\tilde{K}_{0}(1)$.
On the basis of these considerations we conclude that the quadrupole moment of the black hole in our parametrized metric can be measured by fitting the equatorial-plane precession frequency for a circular orbit (43). Quite generically, the latter depends on three coefficients that are fixed asymptotically: $a_{20}, k_{00}$, and $k_{20}\left(w_{20}=0\right.$ to match the results by Ryan [23]). In general relativity, similar coefficients, $M_{2}^{*}$ and $B_{0}$, were introduced in Ref. [24] and are related to these coefficients as

$$
\begin{aligned}
& a_{20}=-3 \frac{M_{2}^{*}}{r_{0}^{3}}=-3 \frac{M_{2}^{*}\left(\epsilon_{0}+1\right)^{3}}{8 M^{3}}, \\
& k_{00}=\frac{M^{2}+4 B_{0}}{r_{0}^{2}}=\left(\epsilon_{0}+1\right)^{2}\left(\frac{1}{4}+\frac{B_{0}}{M^{2}}\right), \\
& k_{20}=0 .
\end{aligned}
$$

In particular, for the Kerr metric one has that

$$
\begin{gathered}
M_{2}^{*}=-\frac{2 M a^{2}}{3} \quad \Longleftrightarrow a_{20}=\frac{2 M a^{2}}{r_{0}^{3}}=\frac{a^{2}\left(\epsilon_{0}+1\right)^{3}}{4 M^{2}}, \\
B_{0}=-\frac{M^{2}-a^{2}}{4} \Longleftrightarrow k_{00}=\frac{a^{2}}{r_{0}^{2}}=\frac{a^{2}\left(\epsilon_{0}+1\right)^{2}}{4 M^{2}} .
\end{gathered}
$$

Note that unlike in [24], the value of $k_{00}$ is fixed here by our coordinate choice. One could choose coordinates such that $k_{00}$ would assume a different value, but this would not alter the final value of $Q$, as it is easy to verify after substituting the corresponding values of $a_{20}$ and $k_{20}$ in (49). As a result, with our choice of coordinates leading to expressions (28), we will always have $k_{00}=a^{2} / r_{0}^{2}$, so that the quadrupole moment is completely determined by the coefficient $a_{20}$.

\section{PARAMETRIZED REPRESENTATION FOR THE ROTATING DILATON BLACK HOLE}

This section is dedicated to the explicit calculation of the coefficients needed for the representation of the parametrized metric that approximate the rotating dilaton black-hole spacetime (6) [19]. This is an important test of our approach and an example of a metric that is more complex than the Kerr solution.

We first substitute (6c)-6e in (18) and taking into account that $A=a$ for a dilaton black hole, we find that

$$
\alpha_{0}(\rho)=0
$$


while from expressions (8) obtain the relations

$$
\begin{aligned}
y & =\mathcal{Y} \\
r^{2} & =\rho(\rho+2 b) \\
N^{2} & =\frac{\left(\sqrt{b^{2}+r^{2}}-b\right)^{2}-2 \mu\left(\sqrt{b^{2}+r^{2}}-b\right)+a^{2}}{r^{2}} \\
W & =\frac{2 a(\mu+b)\left(\sqrt{r^{2}+b^{2}}-b\right)}{r\left(r^{2}+a^{2} y^{2}\right)} \\
B^{2} & =\left(\frac{d \rho}{d r}\right)^{2}=\frac{r^{2}}{b^{2}+r^{2}} \\
K^{2} & =\left(1+\frac{a^{2} y^{2}}{r^{2}}\right)^{-1}\left[\left(1+\frac{a^{2}}{r^{2}}\right)^{2}-\frac{a^{2}\left(1-y^{2}\right)}{r^{2}} N^{2}\right]
\end{aligned}
$$

These expressions coincide up to $\mathcal{O}\left(a^{2}\right)$ with the slowly rotating metric already described in [17]. Furthermore, since $N$ does not depend on $y$, the relation

$$
r_{0}=\sqrt{\left(\mu+b+\sqrt{\mu^{2}-a^{2}}\right)^{2}-b^{2}},
$$

defines the event horizon and reduces to expression (55) of [17] when terms of $\mathcal{O}\left(a^{2}\right)$ are neglected.

By comparing the series expansion of (23) and (56) near spatial infinity (i.e., $x=1$ ) we find that

$$
\begin{aligned}
\epsilon_{0} & =\frac{2 b+2 \mu-r_{0}}{r_{0}}, \\
a_{00} & =\frac{2 b(b+\mu)}{r_{0}^{2}}, \\
b_{00} & =0, \\
w_{00} & =\frac{2 a(b+\mu)}{r_{0}^{2}},
\end{aligned}
$$

which, by comparison with 30 , give correct values for the ADM mass $M$ and the angular momentum $J$, namely

$$
M=\mu+b, \quad J=M a,
$$

and reproduce the same PPN coefficients as for the nonrotating case [17],

$$
\beta=\frac{\mu+2 b}{\mu+b}=1+\frac{b}{M}, \quad \gamma=1 .
$$

We also we find that

$$
a_{20}=\frac{2 a^{2}(\mu+b)}{r_{0}^{3}}=\frac{2 M a^{2}}{r_{0}^{3}},
$$

while all the other asymptotic parameters are zero, except for $k_{00}$, which is given by

$$
k_{00}=\frac{a^{2}}{r_{0}^{2}} .
$$

From expression (49), we find the expected result that the quadrupole moment is given by

$$
Q=-\frac{a_{20} r_{0}^{3}+M\left(k_{00}+k_{20}\right) r_{0}^{2}}{3}=-M a^{2}=-\frac{J^{2}}{M},
$$

for any value of $b$.

Finally, by comparing the series expansion of (23) and (56) near $x=0$ we find that

$$
\begin{aligned}
a_{01}= & \frac{2(\mu+b)\left(2 b^{2}+r_{0}^{2}+\left(2 r_{0}-3 b\right) \sqrt{r_{0}^{2}+b^{2}}\right)}{r_{0}^{2} \sqrt{r_{0}^{2}+b^{2}}} \\
& -3 \frac{r_{0}^{2}+a^{2}}{r_{0}^{2}}, \\
a_{21}= & -\frac{a^{4}+2 a^{2}(\mu+b)\left(b+r_{0}-\sqrt{r_{0}^{2}+b^{2}}\right)}{r_{0}^{4}}, \\
a_{11}= & 0=a_{31}=a_{41}=a_{51}, \ldots, \\
b_{01}= & \frac{r_{0}}{\sqrt{r_{0}^{2}+b^{2}}}-1, \\
b_{11}= & 0=b_{21}=b_{31}=b_{41}=b_{51}, \ldots, \\
w_{01}= & \frac{2 a(\mu+b)\left(b+r_{0}-\sqrt{r_{0}^{2}+b^{2}}\right)}{r_{0}^{3}}, \\
w_{11}= & 0=w_{21}=w_{31}=w_{41}=w_{51}, \ldots, \\
k_{21}= & \frac{a^{4}-2 a^{2}(\mu+b)\left(\sqrt{r_{0}^{2}+b^{2}}-b\right)}{r_{0}^{4}}, \\
k_{01}= & 0=k_{11}=k_{31}=k_{41}=k_{51}, \ldots,
\end{aligned}
$$

and so on. Clearly, $a_{01}, b_{01}$, and $w_{01}$ coincide with the corresponding parameters of the slowly rotating dilaton black hole.

As a final remark, we reinforce a statement already made in Sec. IV] While the line element of the Kerr spacetime can be reproduced exactly within the proposed parametrization with a finite number of terms of the Padé expansion, this does not seem to be possible for the dilaton black hole, whose metric functions are not a ratio of polynomials in powers of $r$, but rather contain square roots of polynomials.

\section{A. Testing the parametrization in the equatorial plane: The binding energy}

Although the parametrization suggested here can be used for generic investigations of the physics of black holes, our main interest is to find a general parametrization for a rotating black-hole spacetime which allows us to model processes connected to electromagnetic emission from matter accreting onto the supermassive black-hole candidate at the center of our Galaxy [26]. Because of this, it is important to test the ability of our parametrization in describing accurately radiative processes near the event horizon and, for instance, the formation of a shadow. This will be the focus of a forthcoming companion paper [28], but some preliminary estimate can already be presented here in terms of the calculation of the simplest basic quantity entering in the physics of accretion flows onto black holes: the binding energy of a particle moving on a circular orbit.

To this scope, we calculate the energy of the particle at the innermost stable circular orbit (ISCO) and thus the binding energy as the amount of energy released by the particle going over from a given stable circular orbit located at $r_{0}$ over to the 


\begin{tabular}{|c|c|c|c|c|}
\hline$b$ & 1st order & 2nd order & 3rd order & exact \\
\hline 0.00 & 5.719095841793664 & 5.719095841793664 & 5.719095841793664 & 5.719095841793664 \\
0.02 & 5.771085348147105 & 5.771085348949989 & 5.771069849415838 & 5.771085349099403 \\
0.20 & 6.207254764244374 & 6.207261486774459 & 6.207262811802919 & 6.207262810978281 \\
0.50 & 6.832236606077545 & 6.832430295600210 & 6.832473869879982 & 6.832473613387891 \\
1.00 & 7.676903064196137 & 7.678772618393546 & 7.679324761207151 & 7.679311080887710 \\
\hline
\end{tabular}

TABLE I. Binding energies as computed at different orders in the radial (continued-fraction) expansion for a nonrotating black hole with a dilaton field $b$. The last column refers to the exact solution.

ISCO at $r_{\text {ISCO }}$, i.e.,

$$
\mathrm{BE}=1-\frac{E\left(r_{\mathrm{ISCO}}\right)}{E\left(r_{0}\right)},
$$

where the initial circular orbit $r_{0}$ is normally considered to be at spatial infinity but needs not be. The binding energy of massive test particles is rather sensitive to the black-hole properties and in Ref. [27] it was calculated for a number of deformed black-hole spacetimes. We here recall that in the case of an extremal Kerr black hole it equals $\simeq 3.8 \%$ for counterrotating particles and $\simeq 42 \%$ for corotating ones, while it is $\simeq 5.7 \%$ for a Schwarzschild black hole. The binding energy also increases above $40 \%$, when the dilaton $b$ ranges from 0 to 1 .

Defining the four-momentum of a massive particle as

$$
p^{\alpha} \equiv m \frac{d x^{\alpha}}{d s},
$$

where $s$ is an invariant affine parameter, we also recall that in a stationary, axisymmetric metric there are three integrals of motion which can be related to the particle's rest mass $m$, to its energy $E=-p_{t}$, and its angular momentum $L=p_{\phi}$. The normalization condition on the four-momentum

$$
p_{\alpha} p^{\alpha}=-m^{2}
$$

leads to the following relation in the equatorial plane

$$
m^{2} g_{r r}\left(\frac{d r}{d s}\right)^{2}=V_{\mathrm{eff}}(r)
$$

where the effective potential is defined as

$$
\begin{aligned}
& V_{\mathrm{eff}}(r) \equiv-\left.\left(g^{t t} E^{2}-2 g^{t \phi} E L+g^{\phi \phi} L^{2}+m^{2}\right)\right|_{\theta=\pi / 2}=(65) \\
& \frac{K^{2}(r, \pi / 2)}{N^{2}(r, \pi / 2)}\left(E-\frac{W(r, \pi / 2)}{K^{2}(r, \pi / 2)} \frac{L}{r}\right)^{2}-\frac{L^{2}}{r^{2} K^{2}(r, \pi / 2)}-m^{2}
\end{aligned}
$$

The energy $E$ and momentum $L$ of a particle on a circular orbit at radial position $r$ can then be determined from the requirements that

$$
V_{\mathrm{eff}}(r)=0, \quad V_{\mathrm{eff}}^{\prime}(r)=0,
$$

where' indicates a derivative in the radial direction. Once the expressions for $L(r)$ and $E(r)$ have been obtained in this way, the position of the ISCO is computed from the additional condition $V_{\text {eff }}^{\prime \prime}(r)=0$ and then to compute the value of the energy at the ISCO $E\left(r_{\text {ISCO }}\right)$, and hence the binding energy as in expression 61 .

Table【reports the binding energies as computed at different orders in the radial (continued-fraction) expansion for a nonrotating black hole with a dilaton field $b$. The last column refers to the exact solution, i.e., to the metric (6). Clearly, the convergence in the spherically symmetric case is excellent and the first order is sufficient to obtain a relative error which is $\sim 10^{-4}$ in the most extreme case of $b=1$. This relative error in the binding energy further reduces to $\sim 10^{-6}$ when considering the expansion at fourth order; clearly these uncertainties are far smaller than what is to be expected from astronomical observations.

The convergent behavior is only slightly less good when rapid rotation is introduced. This is collected in Table II, which refers to a rotating dilaton black hole with $a=0.5 \mu$ (upper rows) and $a=0.95 \mu$ (lower rows), and where the left columns refer to corotating particles, while the right ones to counterrotating particles. In particular, at second order the errors are about $\sim 10^{-4}$ and $\sim 10^{-3}$ and for $a=0.5 \mu, b=1$ and $a=0.95 \mu, b=1$, respectively. We note that as the binding energy of a quasiextreme rotating dilaton black hole reaches its maximum of $42 \%$ for $a \simeq 0.99999 \mu$, reproducing this limiting value with the parametrization would apparently require an expansion up to very high orders in the radial direction. However, since in the equatorial plane the Kerr spacetime (i.e., $b=0$ ) is reproduced exactly already at the first order of the continued fraction, the value of binding energy coincides with the exact binding energy.

\section{PARAMETRIZED REPRESENTATION FOR ROTATING EDGB BLACK HOLES}

While a rotating dilaton black hole corresponds to an essentially Einsteinian theory of gravity with an extra field, a theory containing higher-curvature corrections represents a genuinely non-Einsteinian gravitational theory. In higher than four dimensions, the second order in curvature term (or Gauss-Bonnet term) is the dominating one. In a fourdimensional $(D=4)$ spacetime as the one considered here,

\footnotetext{
${ }^{2}$ We recall that, as already noted in [17] in the slowly rotating regime, the position of the ISCO does not depend on the function $B$.
} 


\begin{tabular}{|c|c|r|r|r|r|r||r|r|r|r|r|}
\hline$a / \mu$ & $b$ & 1st order & 2nd order & 3rd order & 4th order & exact & 1st order & 2nd order & 3rd order & 4th order & exact \\
\hline 0.50 & 0.00 & 8.2118 & 8.2118 & 8.2118 & 8.2118 & 8.2118 & 4.5142 & 4.5142 & 4.5142 & 4.5142 & 4.5142 \\
0.50 & 0.02 & 8.2676 & 8.2675 & 8.2675 & 8.2675 & 8.2675 & 4.5611 & 4.5611 & 4.5611 & 4.5611 & 4.5611 \\
0.50 & 0.20 & 8.7368 & 8.7317 & 8.7318 & 8.7318 & 8.7318 & 4.9555 & 4.9568 & 4.9568 & 4.9568 & 4.9568 \\
0.50 & 0.50 & 9.4142 & 9.3873 & 9.3881 & 9.3881 & 9.3881 & 5.5239 & 5.5315 & 5.5311 & 5.5311 & 5.5311 \\
0.50 & 1.00 & 10.3402 & 10.2568 & 10.2610 & 10.2612 & 10.2613 & 6.2972 & 6.3230 & 6.3217 & 6.3214 & 6.3214 \\
\hline \hline 0.95 & 0.00 & 19.0144 & 19.0144 & 19.0144 & 19.0144 & 19.0144 & 3.8362 & 3.8362 & 3.8362 & 3.8362 & 3.8362 \\
0.95 & 0.02 & 19.0083 & 19.0084 & 19.0084 & 19.0084 & 19.0084 & 3.8791 & 3.8791 & 3.8791 & 3.8791 & 3.8791 \\
0.95 & 0.20 & 19.0140 & 19.0115 & 19.0098 & 19.0097 & 19.0097 & 4.2405 & 4.2430 & 4.2429 & 4.2428 & 4.2428 \\
0.95 & 0.50 & 19.2262 & 19.1424 & 19.1284 & 19.1269 & 19.1270 & 4.7629 & 4.7767 & 4.7757 & 4.7755 & 4.7755 \\
0.95 & 1.00 & 19.9278 & 19.4719 & 19.4191 & 19.4121 & 19.4133 & 5.4763 & 5.5219 & 5.5184 & 5.5170 & 5.5171 \\
\hline
\end{tabular}

TABLE II. The same as in Table \, but for a rotating dilaton black hole with $a=0.5 \mu$ (upper rows) and $a=0.95 \mu$ (lower rows). The left columns refer to corotating particles, while the right ones to counterrotating particles. For all the cases considered, the polar expansion was kept at the order in $\cos ^{2} \theta$ (i.e., at $\cos ^{4} \theta$ ), since higher orders are zero for a dilaton black hole.

the Gauss-Bonnet term is invariant and leads to solutions of the Einstein equations that are not affected unless the dilaton is coupled to the system. Although an exact solution is unknown for such $D=4$ rotating dilaton-Gauss-Bonnet black holes, an approximate metric has been deduced in the regime of slow rotation [25].

In this case the solution has two small parameters

$$
\chi \equiv \frac{a}{M}=\frac{J}{M^{2}}, \quad \zeta \equiv \frac{16 \pi \alpha^{2}}{\beta M^{4}},
$$

where $\alpha$ and $\beta$ are the two coupling constants Einsteindilaton-Gauss-Bonnet theory, with the first one being related to the coupling of higher curvature, while the second one accounts for the coupling with the scalar field. After some algebra, the metric functions up to the order $\mathcal{O}\left(\chi^{3}, \zeta^{2}\right)$ are given by [25]

$$
\begin{aligned}
& f(\rho, \vartheta)=1-\frac{2 M}{\rho}+\frac{M^{2} \chi^{2}}{\rho^{2}}+\zeta\left(-\frac{80 M^{7}}{3 \rho^{7}}+\frac{32 M^{6}}{5 \rho^{6}}+\frac{22 M^{5}}{5 \rho^{5}}+\frac{26 M^{4}}{3 \rho^{4}}+\frac{M^{3}}{3 \rho^{3}}\right)+ \\
& \chi^{2} \zeta\left[-\frac{80 M^{11}}{\rho^{11}}+\frac{7336 M^{10}}{45 \rho^{10}}-\frac{20422 M^{9}}{315 \rho^{9}}+\frac{1917 M^{8}}{245 \rho^{8}}-\right. \\
& \frac{253756 M^{7}}{11025 \rho^{7}}+\frac{838039 M^{6}}{110250 \rho^{6}}-\frac{18551 M^{5}}{5250 \rho^{5}}-\frac{3048 M^{4}}{875 \rho^{4}}-\frac{M^{3}}{6 \rho^{3}}+ \\
& \left.\cos ^{2} \vartheta\left(\frac{240 M^{11}}{\rho^{11}}-\frac{6136 M^{10}}{15 \rho^{10}}+\frac{9214 M^{9}}{21 \rho^{9}}-\frac{26233 M^{8}}{245 \rho^{8}}+\frac{30316 M^{7}}{3675 \rho^{7}}-\frac{1497089 M^{6}}{36750 \rho^{6}}+\frac{11201 M^{5}}{1750 \rho^{5}}+\frac{3019 M^{4}}{875 \rho^{4}}\right)\right] \text {, } \\
& \beta^{2}(\rho, \vartheta)=1-\zeta\left(\frac{48 M^{6}}{\rho^{6}}+\frac{128 M^{5}}{5 \rho^{5}}+\frac{14 M^{4}}{\rho^{4}}+\frac{8 M^{3}}{3 \rho^{3}}+\frac{M^{2}}{\rho^{2}}\right)+ \\
& \chi^{2} \zeta\left[-\frac{720 M^{10}}{\rho^{10}}+\frac{28688 M^{9}}{45 \rho^{9}}+\frac{2102 M^{8}}{15 \rho^{8}}+\frac{616 M^{7}}{5 \rho^{7}}-\frac{907 M^{6}}{45 \rho^{6}}+\frac{139 M^{5}}{15 \rho^{5}}+\frac{5 M^{4}}{\rho^{4}}+\frac{4 M^{3}}{3 \rho^{3}}+\frac{M^{2}}{2 \rho^{2}}+\right. \\
& \left.\cos ^{2} \vartheta\left(\frac{2160 M^{10}}{\rho^{10}}-\frac{26288 M^{9}}{15 \rho^{9}}+\frac{1658 M^{8}}{5 \rho^{8}}-\frac{208 M^{7}}{5 \rho^{7}}+\frac{2767 M^{6}}{15 \rho^{6}}+\frac{11 M^{5}}{\rho^{5}}+\frac{2 M^{4}}{\rho^{4}}\right)\right] \text {, } \\
& \sigma^{2}(\rho, \vartheta)=1+\frac{M^{2} \chi^{2} \cos ^{2} \vartheta}{\rho^{2}}-\chi^{2} \zeta\left(3 \cos ^{2} \vartheta-1\right) Q(\rho), \\
& \omega(\rho, \vartheta)=\frac{2 M^{2} \chi}{\rho^{2}}\left[1+\zeta\left(\frac{40 M^{6}}{3 \rho^{6}}-\frac{24 M^{5}}{5 \rho^{5}}-\frac{3 M^{4}}{\rho^{4}}-\frac{14 M^{3}}{3 \rho^{3}}-\frac{3 M^{2}}{10 \rho^{2}}\right)\right], \\
& \kappa^{2}(\rho, \vartheta)=1+\frac{M^{2} \chi^{2}}{\rho^{2}}+\frac{2 M^{3} \chi^{2} \sin ^{2} \vartheta}{\rho^{3}}-\chi^{2} \zeta\left(3 \cos ^{2} \vartheta-1\right) Q(\rho),
\end{aligned}
$$


where

$$
Q(\rho) \equiv \frac{4463 M^{3}}{2625 \rho^{3}}+\frac{2074 M^{4}}{525 \rho^{4}}+\frac{266911 M^{5}}{36750 \rho^{5}}+\frac{12673 M^{6}}{1575 \rho^{6}}-\frac{12371 M^{7}}{735 \rho^{7}}-\frac{3254 M^{8}}{105 \rho^{8}}-\frac{2536 M^{9}}{45 \rho^{9}}+\frac{80 M^{10}}{\rho^{10}}
$$

The event horizon in these coordinates is a sphere with radius

$$
\rho_{0}=2 M\left(1-\frac{\chi^{2}}{4}-\frac{49 \zeta}{80}-\frac{277 \chi^{2} \zeta}{1920}\right)+\mathcal{O}\left(\chi^{4}, \zeta^{2}\right)
$$

We note that the new coordinates $(A=M \chi)$ are

$$
y=\mathcal{Y}+\mathcal{O}\left(\chi^{2} \zeta\right), \quad r=\rho+\mathcal{O}\left(\chi^{2} \zeta\right),
$$

where the correction $\propto \chi^{2} \zeta$ can be found as a series expansion with respect to $\mathcal{Y}$. Moreover, we have found that $\forall \rho>2 M$, and the following conditions are satisfied

$$
\left|\alpha_{8}(\rho)\right|>\left|\alpha_{6}(\rho)\right|>\left|\alpha_{4}(\rho)\right|
$$

and

$$
\left|\zeta_{8}(\rho)\right|>\left|\zeta_{6}(\rho)\right|>\left|\zeta_{4}(\rho)\right|
$$

which can be interpreted as a signal of the absolute convergence of the series for all $|\mathcal{Y}| \leq 1$.

The relation between the coordinates $(y, r)$ and $(\mathcal{Y}, \rho)$ is given by

$$
\begin{aligned}
y & =\mathcal{Y}\left[1+\chi^{2} \zeta\left(\frac{40 M^{9}}{3 \rho^{9}}-\frac{24 M^{8}}{5 \rho^{8}}-\frac{3 M^{7}}{\rho^{7}}-\frac{14 M^{6}}{3 \rho^{6}}-\frac{3 M^{5}}{10 \rho^{5}}+\mathcal{O}\left(\mathcal{Y}^{2}\right)\right)\right], \\
r^{2} & =\rho\left[1+\chi^{2} \zeta\left(\frac{80 M^{10}}{\rho^{10}}-\frac{3736 M^{9}}{45 \rho^{9}}-\frac{2246 M^{8}}{105 \rho^{8}}-\frac{7961 M^{7}}{735 \rho^{7}}+\frac{27373 M^{6}}{1575 \rho^{6}}+\frac{288961 M^{5}}{36750 \rho^{5}}+\frac{2074 M^{4}}{525 \rho^{4}}+\frac{4463 M^{3}}{2625 \rho^{3}}+\mathcal{O}\left(\mathcal{Y}^{2}\right)\right)\right],
\end{aligned}
$$

so that the location of the horizon in the equatorial plane is given by

$$
r_{0}=2 M\left(1-\frac{\chi^{2}}{4}-\frac{49 \zeta}{80}+\frac{128171 \chi^{2} \zeta}{588000}\right)+\mathcal{O}\left(\chi^{4}, \zeta^{2}\right)
$$

As a result of the coordinate transformations (77) and (78), we obtain

$$
\begin{aligned}
N^{2}=1 & -\frac{2 M}{r}+\frac{M^{2} \chi^{2}}{r^{2}}+\zeta\left(\frac{M^{3}}{3 r^{3}}+\frac{26 M^{4}}{3 r^{4}}+\frac{22 M^{5}}{5 r^{5}}+\frac{32 M^{6}}{5 r^{6}}-\frac{80 M^{7}}{3 r^{7}}\right) \\
& -\chi^{2} \zeta\left(\frac{3267 M^{3}}{1750 r^{3}}+\frac{5017 M^{4}}{875 r^{4}}+\frac{136819 M^{5}}{18375 r^{5}}+\frac{35198 M^{6}}{18375 r^{6}}-\frac{3818 M^{7}}{735 r^{7}}-\frac{4504 M^{8}}{245 r^{8}}+\frac{16 M^{9}}{5 r^{9}}\right) \\
& +\chi^{2} \zeta\left(\frac{3019 M^{4}}{875 r^{4}}+\frac{6388 M^{5}}{875 r^{5}}-\frac{155394 M^{6}}{6125 r^{6}}-\frac{47878 M^{7}}{1225 r^{7}}-\frac{17952 M^{8}}{245 r^{8}}+\frac{2040 M^{9}}{7 r^{9}}-\frac{128 M^{10}}{5 r^{10}}\right) y^{2}+\mathcal{O}\left(\chi^{3}, \zeta^{2}, y^{4}\right), \\
B^{2}= & 1-\zeta\left(\frac{M^{2}}{r^{2}}+\frac{8 M^{3}}{3 r^{3}}+\frac{14 M^{4}}{r^{4}}+\frac{128 M^{5}}{5 r^{5}}+\frac{48 M^{6}}{r^{6}}\right) \\
& +\chi^{2} \zeta\left(\frac{M^{2}}{2 r^{2}}+\frac{4142 M^{3}}{875 r^{3}}+\frac{2949 M^{4}}{175 r^{4}}+\frac{245724 M^{5}}{6125 r^{5}}+\frac{6028 M^{6}}{105 r^{6}}+\frac{12792 M^{7}}{245 r^{7}}-\frac{96 M^{8}}{5 r^{8}}\right) \\
& -\chi^{2} \zeta\left(\frac{4463 M^{3}}{875 r^{3}}+\frac{1724 M^{4}}{175 r^{4}}+\frac{97318 M^{5}}{6125 r^{5}}-\frac{8924 M^{6}}{175 r^{6}}-\frac{43584 M^{7}}{245 r^{7}}-\frac{3056 M^{8}}{7 r^{8}}+\frac{896 M^{9}}{5 r^{9}}\right) y^{2}+\mathcal{O}\left(\chi^{3}, \zeta^{2}, y^{4}\right),
\end{aligned}
$$




$$
\begin{aligned}
W= & \frac{2 M^{2} \chi}{r^{2}}\left[1-\zeta\left(\frac{3 M^{2}}{10 r^{2}}+\frac{14 M^{3}}{3 r^{3}}+\frac{3 M^{4}}{r^{4}}+\frac{24 M^{5}}{5 r^{5}}-\frac{40 M^{6}}{3 r^{6}}\right)\right]+\mathcal{O}\left(\chi^{3}, \zeta^{2}, y^{4}\right) \\
K^{2}= & +\frac{M^{2} \chi^{2}}{r^{2}}+W \frac{M \chi}{r}-\frac{2 M^{3} \chi^{2}}{r^{3}} y^{2} \\
& -\chi^{2} \zeta\left(\frac{4463 M^{3}}{875 r^{3}}+\frac{2074 M^{4}}{175 r^{4}}+\frac{127943 M^{5}}{6125 r^{5}}+\frac{4448 M^{6}}{525 r^{6}}-\frac{2326 M^{7}}{245 r^{7}}-\frac{2792 M^{8}}{35 r^{8}}+\frac{16 M^{9}}{15 r^{9}}\right) y^{2}+\mathcal{O}\left(\chi^{4}, \zeta^{2}, y^{4}\right)
\end{aligned}
$$

Also in this case, by comparing with the series expansion (23) near spatial infinity (i.e., $x=1$ ) we find that

$$
\begin{aligned}
\epsilon_{0} & =\frac{2 M-r_{0}}{r_{0}}, \\
a_{00} & =0=b_{00}, \\
w_{00} & =\frac{2 M^{2} \chi}{r_{0}^{2}}, \\
k_{00} & =\frac{M^{2} \chi^{2}}{r_{0}^{2}},
\end{aligned}
$$

thus implying that the mass, angular momentum, and PPN coefficients of an EDGB black hole obey the same relations as the ones for a Kerr black hole. In addition, it is possible to derive that

$$
\begin{aligned}
a_{20} & =\frac{2 M^{3} \chi^{2}}{r_{0}^{3}}\left(1+\frac{4463}{1750} \zeta\right)+\mathcal{O}\left(\chi^{4}, \zeta^{2}\right) \\
& =\frac{\chi^{2}}{4}\left(1+\frac{61429 \zeta}{14000}\right)+\mathcal{O}\left(\chi^{4}, \zeta^{2}\right),
\end{aligned}
$$

while all of the other asymptotic parameters are zero.

The expression for the quadrupole moment can be derived from expression (49) and yields

$$
\begin{aligned}
Q & =-\frac{a_{20} r_{0}^{3}+M\left(k_{00}+k_{20}\right) r_{0}^{2}}{3} \\
& =-M^{3} \chi^{2}\left(1+\frac{4463}{2625} \zeta\right)+\mathcal{O}\left(\chi^{4}, \zeta^{2}\right),
\end{aligned}
$$

which coincides with the expression for the quadrupole moment found in [25]. We should notice that our result was obtained without the use of ACMC-1 coordinates.

Finally, by comparing the series expansions near the blackhole horizon (i.e., $x=0$ ) we find that

$$
\begin{aligned}
& a_{01}=-\frac{17 \zeta}{60}\left(1-\frac{324899 \chi^{2}}{166600}\right)+\mathcal{O}\left(\chi^{4}, \zeta^{2}\right), \\
& b_{01}=-\frac{361 \zeta}{240}\left(1-\frac{51659 \chi^{2}}{176890}\right)+\mathcal{O}\left(\chi^{4}, \zeta^{2}\right), \\
& w_{01}=-\frac{63 \chi \zeta}{160}+\mathcal{O}\left(\chi^{3}, \zeta^{2}\right) \\
& k_{21}=-\frac{\chi^{2}}{4}\left(1+\frac{438867 \zeta}{49000}\right)+\mathcal{O}\left(\chi^{4}, \zeta^{2}\right),
\end{aligned}
$$

while the other coefficients $a_{i 1}, b_{i 1}$, and $k_{i 1}$ are of order
$\mathcal{O}\left(\chi^{2} \zeta\right)$, e.g.

$$
\begin{aligned}
& a_{21}=\frac{447731}{392000} \chi^{2} \zeta+\mathcal{O}\left(\chi^{4}, \zeta^{2}\right), \\
& b_{21}=\frac{175629}{196000} \chi^{2} \zeta+\mathcal{O}\left(\chi^{4}, \zeta^{2}\right) .
\end{aligned}
$$

In summary, we have shown that it is possible to obtain a full representation of a rotating EDGB black hole within the parametrized-metric approach introduced here. Such a representation is specified by the metric expressions 26, 27) with coefficients given by (81) and (83).

\section{CONCLUSIONS}

We have constructed a parametrization for a general stationary and axisymmetric black hole which could be used for the analysis of physical processes near rotating black holes. Our approach is based on a double expansion in the polar and radial directions of a generic stationary and axisymmetric metric. The polar expansion is handled via the introduction of a series of powers of the elevation from the equatorial plane, i.e., $\cos \theta$ ), while the radial expansion follows the continuedfraction approach in terms of a compactified radial coordinate that has been successfully developed in [17] for a spherically symmetric spacetime.

Since the parametrization uses quite general assumptions about the spacetime of a black hole, such as the presence of Killing vectors along the time and azimuthal coordinates, the absence of closed timelike curves and similar pathologies of the geometry, etc., our approach is essentially independent of any particular metric theory of gravity.

We have shown the validity and effectiveness of our approach by reproducing accurately and with a small number of parameters three relevant and commonly used rotating blackhole spacetimes, namely:

(i) a Kerr black hole, which is reproduced "exactly" in the whole space already at second order in the polar expansion [i.e., at $\mathcal{O}\left(\cos ^{2} \theta\right)$ ] and at first order in the radial expansion;

(ii) a rotating dilaton black hole, which again is reproduced "exactly" at second order in the polar expansion and can be expanded to the desired accuracy with the expansion in the radial direction; 
(iii) a Gauss-Bonnet-dilaton black hole, which is reproduced approximately but at any desired accuracy.

The accuracy of the parametrization has been validated after comparing the values of the binding energy for test particles moving on circular geodesic orbits around a dilaton black hole with the exact ones. Even in the most extreme (and realistic) cases considered, e.g., for a spin of $a=0.95 \mu$ and a dilaton field $b=1$, the relative errors already at the second order are about $\sim 10^{-3}$, and further decrease as the order of the continued fraction is increased. Moreover, even for nearextremal rotation, the convergence of the continued fraction is still very good, being excellent in the equatorial plane, where it is reached already at the few first orders of the Padé approximation.

An important question which we have not addressed here, but that is investigated in detail in a companion paper [28], is about how many orders of the polar and radial expansions are needed for an accurate description of physical processes outside the equatorial plane of the black hole. Although a precise answer obviously depends on the particular spacetime under consideration, some general statements can be made already here. In particular, when considering the shadow cast by various black-hole metrics, we have found that the radial expansion through the Padé approximation is always convergent. Furthermore, the polar expansion leads to the exact solution at the second order for Kerr and dilaton black holes, while higher-order convergence is observed for EDGB black holes and the Johannsen-Psaltis metric. We expect therefore that the parametrized approach presented here will be useful not only to study generic black-hole solutions, but also to interpret the results that will soon be made of the radio emission from the center of our Galaxy.

\section{ACKNOWLEDGMENTS}

It is a pleasure to thank Dimitry Ayzenberg for useful comments on calculation of the quadrupole moment in [25], as well as Ziri Younsi, Yosuke Mizuno, Hector Olivares, and Mariafelicia de Laurentis for numerous discussions. Partial support comes by the ERC Synergy Grant "BlackHoleCam - Imaging the Event Horizon of Black Holes" (Grant No. 610058). A. Z. was also supported by the Alexander von Humboldt Foundation, Germany, Coordenação de Aperfeiçoamento de Pessoal de Nível Superior (CAPES), Brazil, and at the final part by Conselho Nacional de Desenvolvimento Científico e Tecnológico (CNPq). R. K. also acknowledges support from the Alumni Programme of the Alexander von Humboldt Foundation.

\section{Appendix A: Explicit lowest-order metric expression}

We are aware that the derivation of the parametrized metric expressions can appear as intricate. To facilitate the use of our parametrized metrics, we provide here a collection of the explicit expressions of the parametrized metrics for a Kerr, dilaton, and EDGB black hole. In each case we do not necessarily report the highest-order expression of the expansion. Rather, we report the orders that strike a compromise between readability and accuracy. Hence, depending on the various cases, the expressions reported here are either already contained in the main text or are reported here for the first time.

We start with a brief summary of the basic expressions of the metric in terms of the expansion coefficients and how the latter are constrained. Hereafter we will consider the line element (7)

$d s^{2}=-\frac{N^{2}(r, \theta)-W^{2}(r, \theta) \sin ^{2} \theta}{K^{2}(r, \theta)} d t^{2}-2 W(r, \theta) r \sin ^{2} \theta d t d \phi+K^{2}(r, \theta) r^{2} \sin ^{2} \theta d \phi^{2}+\Sigma(r, \theta)\left(\frac{B^{2}(r, \theta)}{N^{2}(r, \theta)} d r^{2}+r^{2} d \theta^{2}\right)$,

assume reflection symmetry across the equatorial plane and neglect coefficients of higher orders. We then find

$$
\begin{aligned}
N^{2}(r, \theta) & =\left(1-\frac{r_{0}}{r}\right)\left[1-\frac{\epsilon_{0} r_{0}}{r}+\frac{\left(a_{00}-\epsilon_{0}+k_{00}\right) r_{0}^{2}}{r^{2}}+\frac{a_{01} r_{0}^{3}}{r^{3}}\right]+\left[\frac{\left(k_{20}+\epsilon_{2}\right) r_{0}^{2}}{r^{2}}+\frac{\left(k_{21}+a_{20}\right) r_{0}^{3}}{r^{3}}+\frac{a_{21} r_{0}^{4}}{r^{4}}\right] \cos ^{2} \theta \\
B(r, \theta) & =1+\frac{b_{00} r_{0}}{r}+\frac{b_{01} r_{0}^{2}}{r^{2}}+\left(\frac{b_{20} r_{0}}{r}+\frac{b_{21} r_{0}^{2}}{r^{2}}\right) \cos ^{2} \theta \\
\Sigma(r, \theta) & =1+\frac{A^{2}}{r^{2}} \cos ^{2} \theta \\
W(r, \theta) & =\frac{1}{\Sigma(r, \theta)}\left[\frac{w_{00} r_{0}^{2}}{r^{2}}+\frac{w_{01} r_{0}^{3}}{r^{3}}+\left(\frac{w_{20} r_{0}^{2}}{r^{2}}+\frac{w_{21} r_{0}^{3}}{r^{3}}\right) \cos ^{2} \theta\right] \\
K^{2}(r, \theta) & =1+\frac{A W(r, \theta)}{r}+\frac{1}{\Sigma(r, \theta)}\left[\frac{k_{00} r_{0}^{2}}{r^{2}}+\left(\frac{k_{20} r_{0}^{2}}{r^{2}}+\frac{k_{21} r_{0}^{3}}{r^{3}}\right) \cos ^{2} \theta\right]
\end{aligned}
$$




\begin{tabular}{|l|c|c|l|}
\hline Parameter(s) & Constrained & Value & Description \\
\hline$\epsilon_{0}, k_{00}, w_{00}$ & asymptotically & $\cdots$ & related to black-hole mass and angular momentum \\
\hline$\epsilon_{2}, b_{20}, w_{20}, k_{20}$ & asymptotically & 0 & for astrophysically realistic black holes \\
\hline$a_{00}, b_{00}$ & asymptotically & 0 & from current PPN estimates \\
\hline$a_{20}$ & asymptotically & $\cdots$ & related to the quadrupole moment \\
\hline$a_{01}$ & event horizon & $\cdots$ & related to the deformation of $g_{t t}$ \\
\hline$a_{21}, k_{21}$ & event horizon & $\cdots$ & related to the deformation of event horizon \\
\hline$w_{01}, w_{21}$ & event horizon & $\cdots$ & related to the rotational deformations of the metric \\
\hline$b_{01}, b_{02}$ & event horizon & $\cdots$ & related to the deformation of $g_{r r}$ \\
\hline
\end{tabular}

TABLE III. Summary of the properties of the various coefficients appearing in the lowest-order expression of the parametrized axisymmetric metric A1 A6. For each coefficient we report the regime where it is constrained, its value (when available), and the physical significance.

(i.e., $\epsilon_{0}, k_{00}, w_{00}, \epsilon_{2}, b_{20}, w_{20}, k_{20}$, and $a_{20}$ ), or by the conditions of the metric near the black-hole event horizon (i.e., $a_{01}$, $a_{21}, k_{21}, w_{01}, w_{21}, b_{01}$, and $\left.b_{21}\right)$. More specifically, the three coefficients $\epsilon_{0}, k_{00}$, and $w_{00}$ can be expressed in terms of radius of the event horizon on the equatorial plane $r_{0}$, of the asymptotic mass $M$, and of the rotation parameter $A$ as

$$
\epsilon_{0}=\frac{2 M-r_{0}}{r_{0}}, \quad k_{00}=\frac{A^{2}}{r_{0}^{2}}, \quad w_{00}=\frac{2 M A}{r_{0}^{2}} .
$$

In order to restore spherical symmetry at large distances at the first PPN order, the coefficients $\epsilon_{0}$ and $b_{20}$ must vanish, while the coefficient $k_{20}$ must vanish to maintain an asymptotic spherical symmetry at the second PPN order, i.e., in order to to have

$$
g_{\phi \phi}=\left(r^{2}+a^{2}\right) \sin ^{2} \theta+\mathcal{O}\left(\frac{1}{r}\right) .
$$

Furthermore, if we exclude the rather exotic situation in which the angular momentum of the black hole depends on the polar angle $\theta$, i.e., on the position of the observer relative to the equatorial plane, then also the coefficient $w_{20}$ must vanish as well. As a result, the following additional conditions can be imposed on the coefficients $\epsilon_{2}, b_{20}, w_{20}$, and $k_{20}$ for astrophysically realistic black-hole solutions (30)

$$
\epsilon_{2}=0=b_{20}=w_{20}=k_{20} \text {. }
$$

Similar considerations apply also to the coefficients $a_{00}$ and $b_{00}$, which must vanish if one wants to match the first-order PPN parameters of general relativity, i.e., if $\beta=\gamma=1$ [cf. Eqs. (30b) and (30c)]. Of course, these coefficients could be taken to be nonzero if more exotic black-hole spacetimes are investigated. Last but surely not least, the coefficient $a_{20}$ is related to the black hole's quadrupole moment and is given (59)

$$
Q=-\frac{a_{20} r_{0}^{3}+M A^{2}}{3}
$$

Next, we turn to the coefficients $a_{01}, a_{21}, b_{01}, b_{21}, k_{21}$, $w_{01}$, and $w_{21}$, which describe the near-horizon behavior of the metric. In particular, the coefficients $a_{21}$ and $k_{21}$ describe deformations of the event horizon and, if the latter is assumed to be spherical, must satisfy the condition

$$
a_{20}+a_{21}+k_{21}=0 .
$$

The coefficients $b_{01}$ and $b_{21}$, on the other hand, correspond to deformations of the $g_{r r}$ metric function and are not expected to play an important role in the dynamics of matter near the event horizon 3 . Finally, the coefficients $w_{01}$ and $w_{21}$ are related to the rotational deformations of the metric, while $a_{01}$ gives the PPN potential of the system. Table III offers a synthetic summary of the various properties of the coefficients, of their values, and how they are constrained.

\section{Parametrized Kerr metric}

We first discuss the explicit form of the parametrized metric for a Kerr black hole as obtained at first order in the radial direction and at second order in the polar direction [i.e., at $\left.\mathcal{O}\left(\cos ^{2} \theta\right)\right]$. After setting $M$ and $A$ to be, respectively, the mass and the specific angular momentum, i.e., $A=a=$ $J / M$, we obtain that the event horizon is defined as

$$
r_{0}=M+\sqrt{M^{2}-a^{2}},
$$

while the asymptotic coefficients have values

$$
\begin{aligned}
& a_{00}=0=b_{00}, \\
& a_{20}=\frac{2 M a^{2}}{r_{0}^{3}} .
\end{aligned}
$$

On the other hand, the strong-field coefficients are given by

$$
\begin{aligned}
& a_{01}=0=w_{01}=w_{21}=b_{01}=b_{21}, \\
& a_{21}=-\frac{a^{4}}{r_{0}^{4}}, \\
& k_{21}=\frac{a^{4}}{r_{0}^{4}}-\frac{2 M a^{2}}{r_{0}^{3}} .
\end{aligned}
$$

\footnotetext{
3 The properties of processes occurring on the equatorial plane, such as those related to the position of ISCO or to the form of the effective potential for particle motion 65, do not depend on the functions $B(r, \theta)$, and thus on the coefficients $b_{01}$ and $b_{21}$.
} 
Using this parametrization it is possible to reproduce the Kerr metric in Boyer-Lindquist coordinates exactly.

\section{Parametrized dilaton black-hole metric}

Next, we turn to the explicit form of the parametrized metric for a rotating dilaton black hole as obtained when truncating at first order in the radial direction and at second order in the polar direction. After setting

$$
\begin{aligned}
M & =\mu+b, \\
A & =a=J / M,
\end{aligned}
$$

the location of the event horizon is given by

$$
r_{0}=\sqrt{\left(\mu+b+\sqrt{\mu^{2}-a^{2}}\right)^{2}-b^{2}},
$$

while the first coefficients are given by

$$
\begin{aligned}
\epsilon_{0} & =\frac{2 b+2 \mu-r_{0}}{r_{0}}, \\
k_{00} & =\frac{a^{2}}{r_{0}^{2}} \\
w_{00} & =\frac{2(b+\mu) a}{r_{0}^{2}} .
\end{aligned}
$$

The asymptotic coefficients are set to be

$$
\begin{aligned}
& a_{00}=\frac{2 b(b+\mu)}{r_{0}^{2}}, \\
& b_{00}=0, \\
& a_{20}=\frac{2 a^{2}(b+\mu)}{r_{0}^{3}},
\end{aligned}
$$

while the strong-field ones are determined to be

$$
\begin{aligned}
a_{01}= & \frac{2(\mu+b)\left[2 b^{2}+r_{0}^{2}+\left(2 r_{0}-3 b\right) \sqrt{r_{0}^{2}+b^{2}}\right]}{r_{0}^{2} \sqrt{r_{0}^{2}+b^{2}}} \\
& -3 \frac{r_{0}^{2}+a^{2}}{r_{0}^{2}}, \\
a_{21}= & -\frac{a^{4}+2 a^{2}(\mu+b)\left(b+r_{0}-\sqrt{r_{0}^{2}+b^{2}}\right)}{r_{0}^{4}}, \\
k_{21}= & \frac{a^{4}-2 a^{2}(\mu+b)\left(\sqrt{r_{0}^{2}+b^{2}}-b\right)}{r_{0}^{4}}, \\
w_{01}= & \frac{2 a(\mu+b)\left(b+r_{0}-\sqrt{r_{0}^{2}+b^{2}}\right)}{r_{0}^{3}}, \\
w_{21}= & 0, \\
b_{01}= & \frac{r_{0}}{\sqrt{r_{0}^{2}+b^{2}}}-1, \\
b_{21}= & 0 .
\end{aligned}
$$

\section{Parametrized EDGB black-hole metric}

Finally, we turn our attention to the parametrization of an EDGB black hole as obtained when truncating at first order in the radial direction and at second order in the polar direction. After setting $M$ to be the mass $A=M \chi$, and taking into account that $\zeta=16 \pi \alpha^{2} /\left(\beta M^{4}\right)$, the position of the event horizon in the equatorial plane is given by

$$
r_{0}=2 M\left(1-\frac{\chi^{2}}{4}-\frac{49 \zeta}{80}+\frac{128171 \chi^{2} \zeta}{588000}\right)+\mathcal{O}\left(\chi^{4}, \zeta^{2}\right)
$$

and depends on $\zeta$. The asymptotic coefficients are given by

$$
a_{00}=b_{00}=0 \text {, }
$$

and by

$$
a_{20}=\frac{\chi^{2}}{4}\left(1+\frac{61429 \zeta}{14000}\right)+\mathcal{O}\left(\chi^{4}, \zeta^{2}\right)
$$

thus implying violation of the Kerr expression for the quadrupole moment [cf. Eq. (82)]. Finally, the strong-field coefficients are found to be

$$
\begin{aligned}
& a_{01}=-\frac{17 \zeta}{60}\left(1-\frac{324899 \chi^{2}}{166600}\right)+\mathcal{O}\left(\chi^{4}, \zeta^{2}\right), \\
& a_{21}=\frac{447731}{392000} \chi^{2} \zeta+\mathcal{O}\left(\chi^{4}, \zeta^{2}\right), \\
& k_{21}=-\frac{\chi^{2}}{4}\left(1+\frac{438867 \zeta}{49000}\right)+\mathcal{O}\left(\chi^{4}, \zeta^{2}\right), \\
& w_{01}=-\frac{63 \chi \zeta}{160}+\mathcal{O}\left(\chi^{3}, \zeta^{2}\right), \\
& w_{21}=\mathcal{O}\left(\chi^{3}, \zeta^{2}\right), \\
& b_{01}=-\frac{361 \zeta}{240}\left(1-\frac{51659 \chi^{2}}{176890}\right)+\mathcal{O}\left(\chi^{4}, \zeta^{2}\right), \\
& b_{21}=\frac{175629}{196000} \chi^{2} \zeta+\mathcal{O}\left(\chi^{4}, \zeta^{2}\right) .
\end{aligned}
$$


[1] P. O. Mazur and E. Mottola Proc. Natl. Acad. Sci. 1019545 (2004).

[2] C. B. M. H. Chirenti and L. Rezzolla, Classical and Quantum Gravity, 24 4191-4206, (2007).

[3] M. A. Abramowicz, W. Kluzniak, and J. P Lasota, Astron. Astrophys. 396 L31-4 (2002).

[4] S. S. Doeleman et al., Nature (London) 455, 78 (2008).

[5] H. Falcke, F. Melia, and E. Agol, Astrophys. J. Lett., 528, L13$16(2000)$.

[6] A. A. Abdujabbarov, L. Rezzolla, B. J. Ahmedov, and Mon. Not. Roy. Astron. Soc. 454, 2423-2435 (2015).

[7] T. Johannsen, D. Psaltis, S. Gillessen, D. P. Marrone, F. Ozel, S. S. Doeleman, and V. L. Fish, Astrophys. J. 758, 30 (2012).

[8] D. Psaltis, N. Wex and M. Kramer, to appear in Astrophys. J. , arXiv: 1510.00394 [astro-ph.HE].

[9] C. Bambi and K. Freese, Phys. Rev. D 79, 043002 (2009).

[10] T. Johannsen and D. Psaltis, Astrophys. J. 716, 187 (2010); Astrophys. J. 718, 446 (2010).

[11] A. E. Broderick, T. Johannsen, A. Loeb, and D. Psaltis, Astrophys. J. 784, 7 (2014).

[12] S. Vigeland, N. Yunes and L. Stein, Phys. Rev. D 83, 104027 (2011).

[13] C. M. Will, Living Rev. Rel. 9, 3 (2006).

[14] T. Johannsen and D. Psaltis, Phys. Rev. D 83, 124015 (2011).

[15] V. Cardoso, P. Pani and J. Rico, Phys. Rev. D 89, 064007 (2014).
[16] E. T. Newman and A. I. Janis, J. Math. Phys. 6, 915 (1965); S. P. Drake and P. Szekeres, Gen. Relativ. Gravit. 32, 445 (2000).

[17] L. Rezzolla and A. Zhidenko, Phys. Rev. D 90, 084009 (2014).

[18] A. Sen, Phys. Rev. Lett. 69, 1006 (1992).

[19] T. Okai, Prog. Theor. Phys. 92, 47 (1994); A. Garcia, D. Galtsov, and O. Kechkin, Phys. Rev. Lett. 74, 1276 (1995).

[20] K. S. Thorne, Rev. Mod. Phys. 52, 299 (1980).

[21] R. P. Geroch, J. Math. Phys. 11, 1955 (1970); J. Math. Phys. 11, 2580 (1970); R. O. Hansen, J. Math. Phys. 15, 46 (1974).

[22] L. Rezzolla and O. Zanotti, Relativistic Hydrodynamics, (Oxford University Press, Oxford, 2013).

[23] F. D. Ryan, Phys. Rev. D 52, 5707 (1995).

[24] G. Pappas and T. A. Apostolatos, arXiv:1211.6299 [gr-qc].

[25] D. Ayzenberg and N. Yunes, Phys. Rev. D 90, 044066 (2014).

[26] http://blackholecam.org

[27] E. P. Esteban and E. Ramos, Phys. Rev. D 38 (1988) 29632971; R. A. Konoplya, Phys. Rev. D 74, 124015 (2006); R. A. Konoplya and Y. C. Liu, Phys. Rev. D 86, 084007 (2012); R. A. Konoplya and A. Zhidenko, Phys. Rev. D 87, 024044 (2013).

[28] Z. Younsi, R. Konoplya, L. Rezzolla, and A. Zhidenko, in preparation (2016). 